\title{
Emotion in the brain: of low roads, high roads and roads less travelled
}

\author{
Beatrice de Gelder*‡, Jack van Honk ${ }^{\S \|}$ and Marco Tamietto*
}

Traditionally, the cerebral cortex is considered the dominant player in understanding human brain functions, with subcortical structures relegated to a subservient role. In a recent Perspective article, Pessoa and Adolphs partly return to this traditional corticocentric view in the field of emotion processing (Emotion processing and the amygdala: from a 'low road' to 'many roads' of evaluating biological significance. Nature Rev. Neurosci. 11, 773-783 (2010))1. In fact, the authors present their 'multiple-waves model' as antithetical to the recent idea of assigning a prominent role to 'low routes' and subcortical structures in non-conscious emotion perception ${ }^{2}$. New hypotheses and critical re-examinations of current theories are always important and welcome, provided that alternative views are not oversimplified. Furthermore, any new model needs to account for the existing evidence and generate novel and testable predictions. In moving beyond our favoured 'low road' proposal, which they refer to as the 'standard' view, Pessoa and Adolphs' Perspective does not fully take stock of currently known facts.

\section{A functional subcortical visual pathway to the amygdala in humans \\ The pathway through the superior colliculus and pulvinar to the amygdala is not simply 'assumed' by analogy from its equivalent in the rodent auditory system, but is actu- ally shown to be functional during non- conscious visual perception of emotional signals in numerous neuroimaging studies that report co-activation among these three regions in healthy subjects and subjects with brain damage ${ }^{2}$. As these findings cannot be dismissed, a convincing explanation of why they might be artefacts is needed along with to disentangle true from false positives.}

\section{Anatomical evidence in primates}

In the tree shrew, a prototypical primate for the anatomical organization of its visual system, projections to the lateral amygdala originate also from the same portions of the pulvinar that receive visual input from the superior colliculus, thereby forming a disynaptic pathway to the amygdala ${ }^{3}$. In humans, using in vivo tractography we have been able to reconstruct the same pathway in the intact brain as well as in the brain of one patient with destruction of the visual cortex ${ }^{2}$. With regard to previous anatomical studies that did not report this pathway, we should remind ourselves that absence of evidence about a phenomenon is not equivalent to evidence that the phenomenon is absent.

\section{Multiple visual pathways}

There are indeed multiple extrageniculostriate anatomical pathways that potentially relay visual information to the amygdala and to other cortical targets, besides the superior colliculus-pulvinar route. Some of these have been linked to specific visual functions, such as motion perception, but not to nonconscious emotion perception. Moreover, there is no evidence that activity in extrastriate cortical areas during non-conscious emotion perception precedes and/or arises independently from modulatory activity in subcortical structures. Therefore, the notion that is defended in the Perspective ${ }^{1}$, that multiple cortical routes - other than the colliculus-pulvinar one - are crucial during non-conscious emotion perception is at present just an assumption.

High and low spatial frequency signals Clearly, explicit discrimination of facial expressions relies on both low and high spatial frequency information, and impairment in processing one of these channels affects emotion recognition. However, during implicit perception of task-irrelevant emotional expressions, low - but not high - spatial frequency information engages the superior colliculus, pulvinar and amygdala ${ }^{4}$. These findings are in keeping with direct physiological evidence that layer $2 \mathrm{~b}$ of the superior colliculus receives visual information from parasol ganglion cells in the retina through magnocellular cells that are preferentially tuned to low spatial frequency information ${ }^{5}$. Why - under the same conditions of implicit perception of low spatial frequency expressions - has the purported activity in parietal and frontal cortices suggested by the authors not been reported in place of activity in the superior colliculus-pulvinar-amygdala pathway?

\section{The amygdala and fear detection}

According to the authors, "the most decisive data" demonstrating that the amygdala is not essential for fear detection come from one single study on patient S.M. with a bilateral amygdala lesion ${ }^{1}$. Surprisingly, however, the opposite - that the human amygdala "may be indispensable to recognize fear in facial expression" - was reported by Adolphs and colleagues in a study on the same patient in 1994 (REF. 6). Ten years later, S.M. still showed a "selective impairment in recognizing fear". The reasons for such an impressive long-range recovery in S.M. are unknown and possibly reflect massive post-lesion or experience-dependent neural plasticity. Nevertheless, S.M.'s recent performance seems inconsistent with most of the studies on patients with amygdala damage $e^{2}$ as well as with previous findings in the same patient.

\section{Speed of reaction and not speed of visual processing} Owing to current methodological limitations, evaluation and comparison of processing speed in cortical and subcortical structures is still an open issue. But independently of this, what is crucial in evolutionary terms is not which brain area starts firing first in response to visual stimulation, but which neural pathway supports quicker access to action and visuomotor integration. Pessoa and Adolphs assume a direct relationship between the latency of neural activity in different brain areas and the latency required by that activity to trigger a behavioural reaction. For example, when measuring speed of involuntary action execution, we found evidence that spontaneous expressive actions are triggered faster when the emotional stimulus is perceived non-consciously rather than consciously ${ }^{7}$.

\section{The role of the cortex}

The cortex, although obviously involved, does not seem necessary for mediating non-conscious emotion processing, basic feelings or reflex-like actions. Patients with total congenital absence of the cerebral cortex can nevertheless exhibit appropriate affective responses ${ }^{8}$. Similarly, the superior colliculus can mediate visuomotor abilities and blindsight in the absence of any cortical contribution in patients who have had a hemispherectomy. Moreover, under conditions requiring a rapid emotional reaction - for example, with rapidly approaching danger - there is a shift of activity from cortical (prefrontal) to subcortical structures (periaqueductal grey) that are directly linked to fight or flight execution ${ }^{9}$. Last, recent 


\section{CORRESPONDENCE}

findings have shown that behavioural and affective flexibility is not an intrinsic property of the orbitofrontal cortex but depends on its interaction with, and its regulation by, the amygdala ${ }^{10,11}$.

Viewed from an anthropocentric perspective, emotions are intrinsically linked to the highest human mental faculties seated in the neocortex. However, from another perspective, emotions are essentially adaptive action dispositions, and phylogenetic continuity is the central heuristic principle of affective neuroscience. Never shall the two perspectives meet?

* Cognitive and Affective Neuroscience Laboratory. Tilburg University, 5000 LE Tilburg, The Netherlands.

${ }^{\ddagger}$ Athinoula A. Martinos Centre for Biomedical Imaging, Massachusetts General Hospital, Harvard Medical School, Charlestown, Massachusetts 02129, USA.

§Department of Experimental Psychology, Utrecht University, Utrecht, The Netherlands.

"Department of Psychiatry and Mental Health, University of Cape Town, South Africa.

Correspondence to B.d.G. and M.T. e-mails:degelder@uvt.nl;M.Tamietto@uvt.nl

doi: 10.1038/nrn2920-c 1
1. Pessoa, L. \& Adolphs, R. Emotion processing and the amygdala: from a 'low road' to 'many roads' of evaluating biological significance. Nature Rev. Neurosci. 11, 773-783 (2010).

2. Tamietto, M. $\&$ de Gelder, B. Neural bases of the nonconscious perception of emotional signals. Nature Rev. Neurosci. 11, 697-709 (2010).

3. Day-Brown, J. D., Wei, H., Chomsung, R. D., Petry, H. M. \& Bickford, M. E. Pulvinar projections to the striatum and amygdala in the tree shrew. Front Neuroanat. 4, 143 (2010).

4. Vuilleumier, P., Armony, J. L., Driver, J. \& Dolan, R. J. Distinct spatial frequency sensitivities for processing faces and emotional expressions. Nature Neurosci. 6 624-631 (2003).

5. Crook, J. D. et al. Y-cell receptive field and collicular projection of parasol ganglion cells in macaque monkey retina. J. Neurosci. 28, 11277-11291 (2008).

6. Adolphs, R., Tranel, D. Damasio, H. \& Damasio, A. Impaired recognition of emotion in facial expressions following bilateral damage to the human amygdala. Nature 372, 669-672 (1994).

7. Tamietto, M. et al. Unseen facial and bodily expressions trigger fast emotional reactions. Proc. Natl Acad. Sci. USA 106, 17661-17666 (2009).

8. Shewmon, D. A., Holmes, G. L. \& Byrne, P. A. Consciousness in congenitally decorticate children developmental vegetative state as self-fulfilling prophecy. Dev. Med. Child Neurol. 41, 364-374 (1999).

9. Mobbs, D. et al. When fear is near: threat imminence elicits prefrontal-periaqueductal gray shifts in humans. Science 317, 1079-1083 (2007).

10. Stalnaker, T. A., Franz, T. M., Singh, T. \& Schoenbaum, G. Basolateral amygdala lesions abolish orbitofrontaldependent reversal impairments. Neuron 54, 51-58 (2007).

11. Williams, L. E., Bargh, J. A., Nocera, C. C. \& Gray, J. R. The unconscious regulation of emotion: nonconscious reappraisal goals modulate emotional reactivity. Emotion 9, 847-854 (2009). 